Path integral quantization of the relativistic dyonium system

This content has been downloaded from IOPscience. Please scroll down to see the full text. 1999 J. Phys. A: Math. Gen. 324199

(http://iopscience.iop.org/0305-4470/32/23/302)

View the table of contents for this issue, or go to the journal homepage for more

Download details:

IP Address: 140.113.38.11

This content was downloaded on 28/04/2014 at 10:22

Please note that terms and conditions apply. 


\title{
Path integral quantization of the relativistic dyonium system
}

\author{
Der-San Chuu and De-Hone Lin \\ Institute of Electro-Physics, National Chiao Tung University, Hsinchu 30043, Taiwan, Republic \\ of China \\ E-mail: d793314@phys.nthu.edu.tw (De-Hone Lin)
}

Received 15 March 1999

Abstract. The relativistic dyonium system is solved by the path integral approach. The energy spectra and the discrete as well as continuous wavefunctions are evaluated explicitly.

\section{Introduction}

Over the past 20 years considerable progress has been made in solving the path integrals (PI) of the non-relativistic potential problems. It is no exaggeration to say that today we are able to solve essentially all path integrals in quantum mechanics, which correspond to problems for which the Schrödinger equation can be solved exactly [1]. However, the same thing cannot be said for the relativistic potential problems. Only a few problems concerning relativistic particles have been discussed using PI. In this paper, we perform the path integral of a relativistic particle with both electric and magnetic charges $\left(e_{1}, g_{1}\right)$ moving in the field created by another charge $\left(e_{2}, g_{2}\right)$ located at the centre, i.e. the dyonium system. This result is an extension of Kleinert's paper [2] where the relativistic Coulomb system was solved using the path integral. The corresponding non-relativistic case of this system was studied by different quantization methods in [3-10].

The method presented in this paper can serve as a prototype for the path integral of arbitrary relativistic spinless systems.

\section{The relativistic path integral}

Adding a vector potential $\boldsymbol{A}(\boldsymbol{x})$ to Kleinert's path integral for a relativistic particle in a scalar potential $V(\boldsymbol{x})$ [2], we find that the path integral representation of the fixed-energy amplitude (Green's function) of a relativistic particle in an external static electromagnetic field is given by [11]

$G\left(\boldsymbol{x}_{b}, \boldsymbol{x}_{a} ; E\right)=\frac{\mathrm{i} \hbar}{2 M c} \int_{0}^{\infty} \mathrm{d} S \int \mathcal{D} \rho(\lambda) \Phi[\rho(\lambda)] \int \mathcal{D}^{3} x(\lambda) \exp \left\{-A_{E} / \hbar\right\} \rho(0)$

with the action

$A_{E}=\int_{\lambda_{a}}^{\lambda_{b}} \mathrm{~d} \lambda\left[\frac{M}{2 \rho(\lambda)} x^{\prime 2}(\lambda)-\mathrm{i}(e / c) \boldsymbol{A}(\boldsymbol{x}) \cdot \boldsymbol{x}^{\prime}(\lambda)-\rho(\lambda) \frac{(E-V(\boldsymbol{x}))^{2}}{2 M c^{2}}+\rho(\lambda) \frac{M c^{2}}{2}\right]$. 
This path integral representation arises from the limiting condition of the $\lambda$-sliced version

$$
\begin{aligned}
G\left(\boldsymbol{x}_{b}, \boldsymbol{x}_{a} ; E\right)= & \lim _{n \rightarrow \infty} \frac{\mathrm{i} \hbar}{2 M c} \int_{0}^{\infty} \mathrm{d} S \prod_{n=1}^{N+1}\left[\int \mathrm{d} \rho_{n} \Phi\left(\rho_{n}\right)\right] \\
& \times \frac{1}{\left(2 \pi \hbar \epsilon_{b} \rho_{b} / M\right)^{3 / 2}} \prod_{n=1}^{N}\left[\int_{-\infty}^{\infty} \frac{\mathrm{d}^{3} x_{n}}{\left(2 \pi \hbar \epsilon_{n} \rho_{n} / M\right)^{D / 2}}\right] \exp \left\{-\frac{1}{\hbar} A^{N}\right\} \rho(0)
\end{aligned}
$$

where the $\lambda$-sliced action

$A^{N}=\sum_{n=1}^{N+1}\left[\frac{M\left(\boldsymbol{x}_{n}-\boldsymbol{x}_{n-1}\right)^{2}}{2 \epsilon_{n} \rho_{n}}-\mathrm{i} \frac{e}{c} \boldsymbol{A}\left(\boldsymbol{x}_{n}\right) \cdot\left(\boldsymbol{x}_{n}-\boldsymbol{x}_{n-1}\right)-\epsilon_{n} \rho_{n} \frac{\left(E-V\left(\boldsymbol{x}_{n}\right)\right)^{2}}{2 M c^{2}}+\epsilon_{n} \rho_{n} \frac{M c^{2}}{2}\right]$

with $\epsilon_{n}=\lambda_{n}-\lambda_{n-1}, \lambda_{b}=\lambda_{N+1}, \lambda_{a}=\lambda_{0}, \boldsymbol{x}_{a}=\boldsymbol{x}\left(\lambda_{0}\right)$ and $\boldsymbol{x}_{b}=\boldsymbol{x}\left(\lambda_{n+1}\right)$.

In equation (2.1), $\hbar / M c$ is the well known Compton wavelength of a particle of mass $M$, $E$ is the system energy and $\boldsymbol{x}$ is the spatial part of the $(3+1)$ vector $x=(\boldsymbol{x}, \tau), S$ is defined as

$$
S=\int_{\lambda_{a}}^{\lambda_{b}} \mathrm{~d} \lambda \rho(\lambda)
$$

where $\rho(\lambda)$ is an arbitrary dimensionless fluctuating scale variable with $\rho(0)$ the terminal point of the function $\rho(\lambda)$, and $\Phi[\rho(\lambda)]$ is some convenient gauge-fixing functional $[2,12,13]$. The only condition on $\Phi[\rho(\lambda)]$ is that

$$
\int \mathcal{D} \rho(\lambda) \Phi[\rho(\lambda)]=1 .
$$

For the dyonium system under consideration, the potential is

$$
V(\boldsymbol{x})=\frac{-e^{2}}{r}
$$

and the vector potential reads

$$
\boldsymbol{A}(\boldsymbol{x})=\hbar q \frac{\left(x_{1} \hat{\boldsymbol{x}}_{2}-x_{2} \hat{\boldsymbol{x}}_{1}\right) x_{3}}{r \boldsymbol{x}_{\perp}^{2}}
$$

where $\boldsymbol{x}_{\perp} \equiv\left(x_{1}, x_{2}, 0\right)$, and $\hat{\boldsymbol{x}}_{i}$ denotes the basis vectors in the Cartesian coordinate frame. The constants $q \equiv-\left(e_{1} g_{2}-e_{2} g_{1}\right) / \hbar c$ and $e^{2} \equiv-e_{1} e_{2}-g_{1} g_{2}$ in equations (2.7) and (2.8) are combinations of the electric and magnetic charges of the two particles, and $r \equiv \sqrt{x_{1}^{2}+x_{2}^{2}+x_{3}^{2}}$ is the radial distance, as usual. The hydrogen atom is a special case of the dyonium system with $e_{1}=-e_{2}=e$ and $q=0$. An electron around a pure magnetic monopole has $e_{1}=e, g_{2}=g, e_{2}=g_{1}=0$. In the vector potential we have taken the gauge freedom $\boldsymbol{A} \rightarrow \boldsymbol{A}(\boldsymbol{x})+\nabla \Lambda(\boldsymbol{x})$ to enforce the transverse gauge $\nabla \cdot \boldsymbol{A}(\boldsymbol{x})=0$. In addition, we have taken advantage of the extra monopole gauge invariance [14] which allows us to choose the shape of the Dirac string that imports the magnetic flux to the monopoles. The field $\boldsymbol{A}(\boldsymbol{x})$ in equation (2.8) has two strings of equal strength importing the flux, one along the positive $x_{3}$-axis from plus infinity to the origin, and the other along the negative $x_{3}$-axis from minus infinity to the origin. As a consequence of monopole gauge invariance, parameter $q$ has to be an integer or a half-integer number [14], a condition referred to as Dirac's charge quantization.

To obtain a tractable path integral for the potential $V(x)=-e^{2} / r$, we choose the fluctuating scale variable $\rho_{n}=r_{n}$ and the gauge-fixing condition

$$
\int \mathcal{D} \rho(\lambda) \Phi[\rho(\lambda)]=\lim _{N \rightarrow \infty} \prod_{n=1}^{N+1}\left[\int \frac{\mathrm{d}\left(x_{4}\right)_{n}}{\left(2 \pi \hbar \epsilon_{n} r_{n} / M\right)^{1 / 2}}\right] \exp \left\{-\frac{1}{\hbar} \sum_{n=1}^{N+1} \frac{M}{2} \frac{\left(\triangle x_{4}\right)_{n}^{2}}{\epsilon_{n} r_{n}}\right\} .
$$


The unity condition is satisfied automatically. With equation (2.9), the path integral of equation (2.3) turns into

$$
\begin{aligned}
G\left(\boldsymbol{x}_{b}, \boldsymbol{x}_{a} ; E\right) & \approx \frac{\mathrm{i} \hbar}{2 M c} \int_{0}^{\infty} \mathrm{d} S \int \mathrm{d}\left(x_{4}\right)_{b} \frac{r_{b}}{\left(2 \pi \hbar \epsilon_{b} r_{b} / M\right)^{2}} \\
& \times \prod_{n=1}^{N}\left[\int_{-\infty}^{\infty} \frac{\mathrm{d}^{4} x_{n}}{\left(2 \pi \hbar \epsilon_{n} r_{n} / M\right)^{2}}\right] \exp \left\{-\frac{1}{\hbar} A^{N}\right\}
\end{aligned}
$$

where the sign $\approx$ in this equation becomes an equality for $N \rightarrow \infty$, and $A^{N}$ is the action of equation (2.4) in which the constant $\rho(0)$ is chosen as $r_{b}$ and the 3-vectors $\boldsymbol{x}_{n}$ of the kinematic term are replaced with the 4-vectors $\vec{x}_{n}$. This extends the kinetic action to

$$
A_{\text {kin }}^{N}=\sum_{n=1}^{N+1} \frac{M}{2} \frac{\left(\vec{x}_{n}-\vec{x}_{n-1}\right)}{\epsilon_{n} r_{n}} .
$$

The path integral can be simplified by the KS transformation [9, 15-17]

$$
\mathrm{d} \vec{x}=2 A(\vec{u}) \mathrm{d} \vec{u} .
$$

The arrow on the top of the $x$ indicates that $x$ has become a 4-vector. For symmetry reasons, the $4 \times 4$ matrix $A(\vec{u})$ is chosen as

$$
A(\vec{u})=\left(\begin{array}{cccc}
u^{3} & u^{4} & u^{1} & u^{2} \\
u^{4} & -u^{3} & -u^{2} & u^{1} \\
u^{1} & u^{2} & -u^{3} & -u^{4} \\
u^{2} & -u^{1} & u^{4} & -u^{3}
\end{array}\right) .
$$

With help of the transformation, the original measure changes into

$$
\mathrm{d}^{4} x=16 r^{2} \mathrm{~d}^{4} u
$$

and the square of the derivative of the 4 -vector $\vec{x}$ has the form

$$
\vec{x}^{\prime 2}=4 \vec{u}^{2} \vec{u}^{\prime 2}=4 r \vec{u}^{\prime 2} \text {. }
$$

The magnetic interaction turns into

$$
\boldsymbol{A} \cdot \boldsymbol{x}^{\prime}=-\frac{\hbar q}{r}\left[\frac{u^{1} u^{\prime 2}-u^{2} u^{\prime 1}}{\left(u^{1}\right)^{2}+\left(u^{3}\right)^{2}}+\frac{u^{4} u^{\prime 3}-u^{3} u^{\prime 4}}{\left(u^{3}\right)^{2}+\left(u^{4}\right)^{2}}\right]\left[\left(u^{1}\right)^{2}+\left(u^{2}\right)^{2}-\left(u^{3}\right)^{2}-\left(u^{4}\right)^{2}\right] \text {. }
$$

We obtain a path integral in the continuum limit equivalent to equation (2.10),

$$
G\left(\boldsymbol{x}_{b}, \boldsymbol{x}_{a} ; E\right)=\frac{\mathrm{i} \hbar}{2 M c} \int_{0}^{\infty} \mathrm{d} S \mathrm{e}^{S E e^{2} / \hbar M c^{2}} G\left(\vec{u}_{b}, \vec{u}_{a} ; S\right)
$$

where $G\left(\vec{u}_{b}, \vec{u}_{a} ; S\right)$ denotes the amplitude

$$
G\left(\vec{u}_{b}, \vec{u}_{a} ; S\right)=\frac{1}{16} \int \frac{\mathrm{d}\left(x_{4}\right)_{b}}{r_{b}} \int \mathcal{D}^{4} u(\lambda) \exp \left\{\frac{1}{\hbar} A\right\}
$$

with the action

$$
A=\int_{0}^{S} \mathrm{~d} \lambda\left[\frac{m \vec{u}^{\prime 2}}{2}-\mathrm{i}\left(\vec{A} \cdot \vec{u}^{\prime}(\lambda)\right)+\frac{m \omega^{2} \vec{u}^{2}}{2}-\frac{4 \hbar^{2} \alpha^{2}}{2 m \vec{u}^{2}}\right]
$$

and the functional measure in the $\lambda$-sliced form

$$
\int D^{4} u(\lambda) \approx \frac{1}{\left(2 \pi \hbar \epsilon_{b} / m\right)^{2}} \prod_{n=1}^{N}\left[\int_{-\infty}^{\infty} \frac{\mathrm{d}^{4} u_{n}}{\left(2 \pi \hbar \epsilon_{n} / m\right)^{2}}\right]
$$


The parameters are

$$
m=4 M \quad \omega^{2}=\frac{M^{2} c^{4}-E^{2}}{4 M^{2} c^{2}} .
$$

Here $\alpha$ denotes the 'fine'-structure constant $\alpha \equiv e^{2} / \hbar c$, and $\vec{A}_{n} \cdot \vec{u}^{\prime}$ is given by equation (2.16).

Let us analyse the effect which comes from the magnetic interaction in the Coulomb system. We first express $\left(u^{1}, u^{2}, u^{3}, u^{4}\right)$ in terms of Euler angles (e.g. [14]):

$$
\left.\begin{array}{l}
u^{1}=\sqrt{r} \cos (\theta / 2) \cos [(\varphi+\gamma) / 2] \\
u^{2}=\sqrt{r} \cos (\theta / 2) \sin [(\varphi+\gamma) / 2] \\
u^{3}=\sqrt{r} \sin (\theta / 2) \cos [(\varphi-\gamma) / 2] \\
u^{4}=\sqrt{r} \sin (\theta / 2) \sin [(\varphi-\gamma) / 2]
\end{array}\right\}\left(\begin{array}{c}
0 \leqslant \theta \leqslant \pi \\
0 \leqslant \varphi \leqslant 2 \pi \\
0 \leqslant \gamma \leqslant 4 \pi
\end{array}\right) .
$$

Then the action of equation (2.19) reads

$$
\begin{aligned}
A=\int_{0}^{S} \mathrm{~d} \lambda\left\{\frac{m}{2}\left[u^{\prime 2}+\frac{u^{2}}{4}\left(\theta^{\prime 2}+\varphi^{\prime 2}+\gamma^{\prime 2}+2 \varphi^{\prime}\left(\gamma^{\prime}-\frac{4 \hbar q \mathrm{i}}{m u^{2}}\right) \cos \theta\right)\right]\right. \\
\left.+\frac{m \omega^{2} \vec{u}^{2}}{2}-\frac{4 \hbar^{2} \alpha^{2}}{2 m \vec{u}^{2}}\right\} .
\end{aligned}
$$

In terms of canonical momenta the coordinates $(u, \theta, \varphi, \gamma)$ can be as follows:

$$
\begin{aligned}
u^{\prime} & =\frac{p_{u}}{m} \\
\theta^{\prime} & =\frac{p_{\theta}}{\xi} \\
\gamma^{\prime} & =\frac{1}{\xi \sin ^{2} \theta}\left[p_{\gamma}+\eta \xi \cos ^{2} \theta-p_{\varphi} \cos \theta\right] \\
\varphi^{\prime} & =\frac{1}{\xi \sin ^{2} \theta}\left[p_{\varphi}-\eta \xi \cos \theta-p_{\gamma} \cos \theta\right]
\end{aligned}
$$

where the variables $\eta \equiv-4 \hbar q \mathrm{i} / m u^{2}$ and $\xi \equiv m u^{2} / 4$. Since equation (2.22) transforms $\mathrm{d} x_{4}$ into

$$
\begin{aligned}
\mathrm{d} x_{4} & =2\left(u^{2} \mathrm{~d} u^{1}-u^{2} \mathrm{~d} u^{1}+u^{4} \mathrm{~d} u^{3}-u^{3} \mathrm{~d} u^{4}\right) \\
& =r(\cos \theta \mathrm{d} \varphi+\mathrm{d} \gamma)
\end{aligned}
$$

the integral $\int\left(\mathrm{d} x_{4}\right)_{b} / r_{b}$ in the measure of equation (2.18) becomes $\int \mathrm{d} \gamma_{b}$. This is due to the $\boldsymbol{x}$ and thus the angles $(\theta, \varphi)$ remain fixed during the $x_{4}$ integration. With the help of equation (2.24), we obtain the canonical form of the path integral,

$\frac{\mathrm{i} \hbar}{2 M c} \int_{0}^{\infty} \mathrm{d} S \mathrm{e}^{S E e^{2} / \hbar M c^{2}} \frac{1}{16} \int_{0}^{4 \pi} \mathrm{d} \gamma_{b} \int \mathcal{D}^{4} u(\lambda) \int_{-\infty}^{\infty} \frac{\mathcal{D}^{4} p(\lambda)}{2 \pi \hbar} \exp \left\{-\frac{1}{\hbar} A\right\}$

where the action is given by

$$
A=\int_{0}^{S} \mathrm{~d} \lambda\left\{-\mathrm{i}\left[p_{u} u^{\prime}+p_{\theta} \theta^{\prime}+p_{\varphi} \varphi^{\prime}+p_{\gamma} \gamma^{\prime}\right]+H\right\}
$$

with the Hamiltonian

$$
\begin{aligned}
H=\frac{1}{2 m}\left\{p_{u}^{2}\right. & \left.+\frac{4}{\vec{u}^{2}}\left[p_{\theta}^{2}+\frac{1}{\sin ^{2} \theta}\left(p_{\varphi}^{2}+p_{\gamma}^{2}-2\left(p_{\gamma}+\hbar q\right) p_{\varphi} \cos \theta\right)\right]\right\} \\
& +\frac{4}{2 m \vec{u}^{2}}\left[-2 \hbar q p_{\gamma}+\hbar^{2}\left(\alpha^{2}+q^{2}\right)\right]+\frac{1}{2} m \omega^{2} \vec{u}^{2} .
\end{aligned}
$$


In the canonical path integral, the momenta are dummy integration variables so that we can replace $\left(p_{\gamma}+\hbar q\right)$ by $p_{\gamma}$. Then the action becomes

$$
A=\int_{0}^{S} \mathrm{~d} \lambda\left\{-\mathrm{i}\left[p_{u} u^{\prime}+p_{\theta} \theta^{\prime}+p_{\varphi} \varphi^{\prime}+\left(p_{\gamma}-\hbar q\right) \gamma^{\prime}\right]+\bar{H}\right\}
$$

with the Hamiltonian

$$
\begin{aligned}
\bar{H}=\frac{1}{2 m}\left\{p_{u}^{2}\right. & \left.+\frac{4}{\vec{u}^{2}}\left[p_{\theta}^{2}+\frac{1}{\sin ^{2} \theta}\left(p_{\varphi}^{2}+p_{\gamma}^{2}-2 p_{\gamma} p_{\varphi} \cos \theta\right)\right]\right\} \\
& +\frac{4}{2 m \vec{u}^{2}}\left[-2 \hbar q\left(p_{\gamma}-\hbar q\right)+\hbar^{2}\left(\alpha^{2}+q^{2}\right)\right]+\frac{m \omega^{2} \vec{u}^{2}}{2} .
\end{aligned}
$$

This differs from the pure relativistic Coulomb system in two ways [2].

First, the Hamiltonian has an extra centrifugal barrier proportional to the charge parameter $q$ :

$$
V(r)=\frac{-2 \hbar q\left(p_{\gamma}-\hbar q\right)}{2 M r}
$$

Secondly, the action of equation (2.29) contains an additional term

$$
\triangle A=-\hbar q \int_{0}^{S} \mathrm{~d} \lambda \gamma^{\prime}
$$

Fortunately, this is a pure surface term $\triangle A=-\hbar q\left(\gamma_{b}-\gamma_{a}\right)$. The modification consists of a simple extra phase factor in the integral over $\gamma_{b}$ so that

$$
G\left(\boldsymbol{x}_{b}, \boldsymbol{x}_{a} ; E\right)=\frac{\mathrm{i} \hbar}{2 M c} \int_{0}^{\infty} \mathrm{d} S \mathrm{e}^{S E e^{2} / \hbar M c^{2}} \frac{1}{16} \int_{0}^{4 \pi} \mathrm{d} \gamma_{b} \mathrm{e}^{-\mathrm{i} q\left(\gamma_{b}-\gamma_{a}\right)} G\left(\vec{u}_{b}, \vec{u}_{a} ; S\right) .
$$

Since the integral over $\gamma_{b}$ forces the momentum $p_{r}$ in the canonical action (2.29) to take the value $\hbar q$. This eliminates the term proportional to $p_{\gamma}-\hbar q$ in equation (2.30), therefore the fixed-energy amplitude in $u$-space turns into

$$
G\left(\vec{u}_{b}, \vec{u}_{a} ; S\right)=\left[\int_{-\infty}^{\infty} \mathcal{D}^{4} u(\lambda)\right] \exp \left\{-\frac{1}{\hbar} A\right\}
$$

with the action

$$
A=\int_{0}^{S} \mathrm{~d} \lambda\left\{\frac{m \vec{u}^{\prime 2}}{2}+\frac{m \omega^{2} \vec{u}^{2}}{2}-\frac{4\left(\alpha^{2}+q^{2}\right) \hbar^{2}}{2 m \vec{u}^{2}}\right\} .
$$

It describes a particle with mass $m=4 M$ moving as a function of the 'pseudo-time' $\lambda$ in a four-dimensional harmonic oscillator potential of frequency

$$
\omega^{2}=\frac{M^{2} c^{4}-E^{2}}{4 M^{2} c^{2}} .
$$

The oscillator possesses an additional attractive potential $-4\left(\alpha^{2}+q^{2}\right) \hbar^{2} / 2 m \vec{u}^{2}$ which is conveniently parametrized in the form of a centrifugal barrier

$$
V_{\text {extra }}=\hbar^{2} \frac{l_{\text {extra }}^{2}}{2 m \vec{u}^{2}}
$$

whose squared angular momentum has the negative value $l_{\text {extra }}^{2} \equiv-4\left(\alpha^{2}+q^{2}\right)$. There are no $\lambda$-slicing corrections. This is ensured by the affine connection of KS transformation 
satisfying $\Gamma_{\mu}^{\mu \lambda}=g^{\mu v} e_{i}{ }^{\lambda} \partial_{\mu} e^{i}{ }_{\nu}=0$ and the transverse gauge $\partial_{i} A^{i}=0$ [16]. The path integral equation (2.35) can be performed and is given by

$G\left(\vec{u}_{b}, \vec{u}_{a} ; S\right)=\frac{1}{u_{b} u_{a}} \sum_{l=0}^{\infty} G\left(u_{b}, u_{a} ; S, l\right) \frac{l+1}{2 \pi^{2}} \sum_{k_{1}, k_{2}=-l / 2}^{l / 2} d_{k_{1}, k_{2}}^{l / 2}\left(\theta_{b}\right) d_{k_{1}, k_{2}}^{l / 2}\left(\theta_{a}\right) \mathrm{e}^{\mathrm{i} k_{1}\left(\varphi_{b}-\varphi_{a}\right)+\mathrm{i} k_{2}\left(\gamma_{b}-\gamma_{a}\right)}$

with the radial amplitude

$G\left(u_{b}, u_{a} ; S, l\right)=\frac{m \omega}{\hbar \sinh \omega S} \mathrm{e}^{-(m \omega / 2 \hbar)\left(u_{b}^{2}+u_{a}^{2}\right) \operatorname{coth} \omega S} I_{\sqrt{(l+1)^{2}-4\left(\alpha^{2}+q^{2}\right)}}\left(\frac{m}{\hbar} \frac{\omega u_{b} u_{a}}{\sinh \omega S}\right)$

where $d_{k_{1}, k_{2}}^{l / 2}(\theta) \mathrm{e}^{\mathrm{i} k_{1} \varphi+\mathrm{i} k_{2} \gamma}$ are the representation functions of the rotation group (e.g. [16]). The integral $\int_{0}^{4 \pi} \mathrm{d} \gamma_{a} \mathrm{e}^{-\mathrm{i} q\left(\gamma_{b}-\gamma_{a}\right)}$ in equation (2.33) can be easily done. We arrive at the fixed-energy amplitude of the relativistic dyonium system, now labelled by the subscript $D$,

$G\left(\boldsymbol{x}_{b}, \boldsymbol{x}_{a} ; E_{D}\right)=\frac{1}{\sqrt{r_{b} r_{a}}} \sum_{l_{D}} G\left(r_{b}, r_{a} ; E_{D}, l_{D}\right) \sum_{k=-l_{D}}^{l_{D}} Y_{l_{D}, k, q}\left(\theta_{b}, \varphi_{b}\right) Y_{l_{D}, k, q}^{\star}\left(\theta_{a}, \varphi_{a}\right)$

where $Y_{l_{D}, k, q}\left(\theta_{b}, \varphi_{b}\right)$ are the so-called monopole harmonics

$$
Y_{l_{D}, k, q}(\theta, \varphi)=\sqrt{\frac{l+1}{4 \pi}} \mathrm{e}^{\mathrm{i} k \varphi} d_{k, q}^{l_{D}}(\theta)
$$

and $l_{D}$ is defined as $l / 2$. The radial amplitude for the dyonium is

$$
\begin{aligned}
& G\left(r_{b}, r_{a} ; E_{D}, l_{D}\right)=\frac{\mathrm{i} \hbar}{2 M c} \frac{1}{2} \int_{0}^{\infty} \mathrm{d} S \mathrm{e}^{S E_{D} e^{2} / \hbar M c^{2}} \\
& \times \frac{m \omega}{\hbar \sinh \omega S} \mathrm{e}^{-(m \omega / 2 \hbar)\left(r_{b}+r_{a}\right) \operatorname{coth} \omega S} I_{\sqrt{\left(2 l_{D}+1\right)^{2}-4\left(\alpha^{2}+q^{2}\right)}}\left(\frac{m \omega \sqrt{r_{b} r_{a}}}{\hbar \sinh \omega S}\right) .
\end{aligned}
$$

This integral can be calculated by employing the formula

$$
\begin{array}{r}
\int_{0}^{\infty} \mathrm{d} y \frac{\mathrm{e}^{2 v y}}{\sinh y} \exp \left[-\frac{1}{2} t\left(\zeta_{a}+\zeta_{b}\right) \operatorname{coth} y\right] I_{\mu}\left(\frac{t \sqrt{\zeta_{b} \zeta_{a}}}{\sinh y}\right) \\
=\frac{\Gamma((1+\mu) / 2-v)}{t \sqrt{\zeta_{b} \zeta_{a}} \Gamma(\mu+1)} W_{v, \mu / 2}\left(t \zeta_{b}\right) M_{v, \mu / 2}\left(t \zeta_{b}\right)
\end{array}
$$

with the range of validity

$$
\begin{aligned}
& \zeta_{b}>\zeta_{a}>0 \\
& \operatorname{Re}[(1+\mu) / 2-v]>0 \\
& \operatorname{Re}(t)>0 \quad|\arg t|<\pi
\end{aligned}
$$

where $M_{\mu, \nu}$ and $W_{\mu, \nu}$ are the Whittaker functions [18] (see $\mathrm{p}$ 1087). Thus we complete the integration of equation (2.42), and find the amplitude for $r_{b}>r_{a}$ in the closed form

$$
\begin{aligned}
& G\left(r_{b}, r_{a} ; E_{D}, l_{D}\right)=\frac{\mathrm{i} \hbar}{2 M c} \frac{M c}{\sqrt{M^{2} c^{4}-E_{D}^{2}}} \\
& \times \frac{\Gamma\left(\frac{1}{2}+\frac{1}{2} \sqrt{\left(2 l_{D}+1\right)^{2}-4\left(\alpha^{2}+q^{2}\right)}-E_{D} \alpha / \sqrt{M^{2} c^{4}-E_{D}^{2}}\right)}{\sqrt{r_{b} r_{a}} \Gamma\left(\sqrt{\left(2 l_{D}+1\right)^{2}-4\left(\alpha^{2}+q^{2}\right)}+1\right)} \\
& \times W_{E_{D} \alpha / \sqrt{M^{2} c^{4}-E_{D}^{2}}}, \sqrt{\left(2 l_{D}+1\right)^{2}-4\left(\alpha^{2}+q^{2}\right) / 2}\left(\frac{2}{\hbar c} \sqrt{M^{2} c^{4}-E_{D}^{2}} r_{b}\right) \\
& \times M_{E_{D} \alpha / \sqrt{M^{2} c^{4}-E_{D}^{2}}, \sqrt{\left(2 l_{D}+1\right)^{2}-4\left(\alpha^{2}+q^{2}\right) / 2}}\left(\frac{2}{\hbar c} \sqrt{M^{2} c^{4}-E_{D}^{2}} r_{a}\right) \text {. }
\end{aligned}
$$


The energy spectra and wavefunctions can be extracted from the poles of equation (2.44). For convenience, we define the following variables:

$$
\begin{aligned}
\kappa & =\frac{1}{\hbar c} \sqrt{M^{2} c^{4}-E_{D}^{2}} \\
v & =\frac{\alpha E_{D}}{\sqrt{M^{2} c^{4}-E_{D}^{2}}} \\
\tilde{l}_{D} & =\sqrt{\left(l_{D}+1 / 2\right)^{2}-\left(\alpha^{2}+q^{2}\right)}-\frac{1}{2} .
\end{aligned}
$$

From the poles of $G\left(r_{b}, r_{a} ; E_{D}, l_{D}\right)$, we find that the energy levels must satisfy the equality

$$
-v+\tilde{l}_{D}+1=-n_{r} \quad n_{r}=0,1,2,3, \ldots
$$

After some mathematical manipulation, we have

$$
E_{n_{r}, l_{D}}= \pm M c^{2}\left[1+\frac{\alpha^{2}}{\left(\frac{1}{2}+\frac{1}{2} \sqrt{\left(2 l_{D}+1\right)^{2}-4\left(q^{2}+\alpha^{2}\right)}+n_{r}\right)^{2}}\right]^{-1 / 2} .
$$

The pole positions, which satisfy $v=\tilde{n}_{l_{D}} \equiv n+\tilde{l}_{D}-l_{D}\left(n=l_{D}+1, l_{D}+2, l_{D}+3, \ldots\right)$, correspond to the bound states of the relativistic dyonium system. Near the positive-energy poles, we use the behaviour for $v \approx \tilde{n}_{l_{D}}$,

$$
\Gamma\left(-v+\tilde{l}_{D}+1\right) \frac{M}{\hbar \kappa} \approx-\frac{(-)^{n_{r}}}{\tilde{n}_{l_{D}}^{2} n_{r} !} \frac{1}{\tilde{a}_{D}}\left(\frac{E_{D}}{M c^{2}}\right)^{2} \frac{2 \hbar M c^{2}}{E_{D}^{2}-E_{n l_{D}}^{2}}
$$

where $\tilde{a}_{D} \equiv a_{D} M c^{2} / E_{D}\left(a_{D}=\hbar^{2} / M e^{2}\right)$ is the modified energy-dependent dyonium Bohr radius and $n \equiv n_{r}+l_{D}+1$ is the principal quantum number, to extract the wavefunctions of the dyonium system

$$
\begin{aligned}
& G\left(r_{b}, r_{a} ; E_{D}, l_{D}\right)=-\frac{\mathrm{i} \hbar}{2 M c} \frac{1}{\left(r_{b} r_{a}\right)} \sum_{n=l_{D}+1}^{\infty}\left(\frac{E_{D}}{M c^{2}}\right)^{2} \frac{2 \hbar M c^{2}}{E_{D}^{2}-E_{n l_{D}}^{2}} \\
& \times \frac{1}{\left[\left(2 \tilde{l}_{D}+1\right) !\right]^{2}} \frac{1}{\tilde{n}_{l_{D}}^{2} \tilde{a}_{D}} \frac{\left(\tilde{n}_{l_{D}}+\tilde{l}_{D}\right) !}{\left(n-l_{D}-1\right) !} \mathrm{e}^{-\left(r_{b}+r_{a}\right) / \tilde{a}_{D} \tilde{n}_{l_{D}}}\left(\frac{2 r_{b}}{\tilde{a}_{D} \tilde{n}_{l_{D}}} \frac{2 r_{a}}{\tilde{a}_{D} \tilde{n}_{l_{D}}}\right)^{\tilde{l}_{D}+1} \\
& \times M\left(-n+l_{D}+1,2 \tilde{l}_{D}+2 ; \frac{2 r_{b}}{\tilde{a}_{D} \tilde{n}_{l_{D}}}\right) M\left(-n+l_{D}+1,2 \tilde{l}_{D}+2 ; \frac{2 r_{a}}{\tilde{a}_{D} \tilde{n}_{l_{D}}}\right) \\
& =-\frac{\mathrm{i} \hbar}{2 M c} \frac{1}{\left(r_{b} r_{a}\right)} \sum_{n=l_{D}+1}^{\infty}\left(\frac{E_{D}}{M c^{2}}\right)^{2} \frac{2 \hbar M c^{2}}{E_{D}^{2}-E_{n l_{D}}^{2}} R_{n l_{D}}\left(r_{b}\right) R_{n l_{D}}^{*}\left(r_{a}\right)+\cdots
\end{aligned}
$$

where we have expressed the Whittaker function $M_{\lambda, \mu}(z)$ in terms of the Kummer functions $M(a, b ; z)[18](\mathrm{p} 1087)$

$$
M_{\lambda, \mu}(z)=z^{\mu+1 / 2} \mathrm{e}^{-z / 2} M\left(\mu-\lambda+\frac{1}{2}, 2 \mu+1 ; z\right) .
$$

From this we obtain the radial wavefunctions

$$
\begin{aligned}
R_{n l_{D}}(r)=\frac{1}{\tilde{n}_{l_{D}} \tilde{a}_{D}^{1 / 2}} \frac{1}{\left(2 \tilde{l}_{D}+1\right) !} \sqrt{\frac{\left(\tilde{n}_{l_{D}}+\tilde{l}_{D}\right) !}{\left(n-l_{D}-1\right) !}} \\
\\
\quad \times\left(\frac{2 r}{\tilde{a}_{D} \tilde{n}_{l_{D}}}\right)^{\tilde{l}_{D^{+1}}} \mathrm{e}^{-r / \tilde{a}_{D} \tilde{n}_{l_{D}} M\left(-n+l_{D}+1,2 \tilde{l}_{D}+2 ; \frac{2 r}{\tilde{a}_{D} \tilde{n}_{l_{D}}}\right) .}
\end{aligned}
$$


The normalized wavefunctions are given by

$$
\Psi_{n l_{D} k q}(\boldsymbol{x})=\frac{1}{r} R_{n l_{D}}(r) Y_{l_{D}, k, q}(\hat{\boldsymbol{x}}) .
$$

Before extracting the continuous wavefunction we note that the parameter $\kappa$ is real for $\left|E_{D}\right|<M c^{2}$. For $\left|E_{D}\right|>M c^{2}$, the square root in equation (2.45) has two imaginary solutions

$$
\kappa=\mp \mathrm{i} \tilde{k} \quad \tilde{k}=\frac{1}{\hbar c} \sqrt{E_{D}^{2}-M^{2} c^{4}}
$$

corresponding to

$$
v= \pm \mathrm{i} \tilde{v} \quad \tilde{v}=\frac{E_{D} \alpha}{\hbar c \tilde{k}} .
$$

Therefore, the amplitude has a right-handed cut for $E_{D}>M c^{2}$ and $E_{D}<-M c^{2}$. For simplicity, we will only consider the positive energy cut.

The continuous wavefunctions are recovered from the discontinuity of the amplitudes $G\left(r_{b}, r_{a} ; E_{D}, l_{D}\right)$ across the cut in the complex $E_{D}$-plane. Hence we have

$$
\begin{aligned}
\operatorname{disc} G\left(r_{b}, r_{a} ;\right. & \left.E_{D}>M c^{2}, l_{D}\right)=G\left(r_{b}, r_{a} ; E_{D}+\mathrm{i} \eta, l_{D}\right)-G\left(r_{b}, r_{a} ; E_{D}-\mathrm{i} \eta, l_{D}\right) \\
= & -\frac{\mathrm{i} \hbar}{2 M c} \frac{1}{\left(r_{b} r_{a}\right)} \frac{M}{\hbar \tilde{k}} \\
& \times\left[\frac{\Gamma\left(-\mathrm{i} \tilde{v}+\tilde{l}_{D}+1\right)}{\left(2 \tilde{l}_{D}+1\right) !} W_{\mathrm{i} \tilde{v}, \tilde{l}_{D}+1 / 2}\left(-2 \mathrm{i} \tilde{k} r_{b}\right) M_{\mathrm{i} \tilde{v}, \tilde{l}_{D}+1 / 2}\left(-2 \mathrm{i} \tilde{k} r_{a}\right)+(\tilde{v} \rightarrow-\tilde{v})\right] .
\end{aligned}
$$

Using the relations [16]

$$
M_{\kappa, \mu}(z)=\mathrm{e}^{ \pm \mathrm{i} \pi(2 \mu+1) / 2} M_{-\kappa, \mu}(-z)
$$

where the sign is positive or negative depending on whether $\operatorname{Im} z>0$ or $\operatorname{Im} z<0$, and [18] (p 1090),

$$
\begin{aligned}
W_{\lambda, \mu}(z)=\mathrm{e}^{\mathrm{i} \pi \lambda} & \mathrm{e}^{-\mathrm{i} \pi(\mu+1 / 2)} \frac{\Gamma\left(\mu+\lambda+\frac{1}{2}\right)}{\Gamma(2 \mu+1)} \\
\times & {\left[M_{\lambda, \mu}(z)-\frac{\Gamma(2 \mu+1)}{\Gamma\left(\mu-\lambda+\frac{1}{2}\right)} \mathrm{e}^{-\mathrm{i} \pi \lambda} W_{-\lambda, \mu}\left(\mathrm{e}^{-\mathrm{i} \pi} z\right)\right] }
\end{aligned}
$$

which is valid only for $\arg (z) \in(-\pi / 2,3 \pi / 2)$ and $2 \mu \neq-1,-2,-3, \ldots$ The discontinuity of the amplitude is found to be

$$
\begin{aligned}
\operatorname{disc} G\left(r_{b}, r_{a} ;\right. & \left.E_{D}>M c^{2}, l_{D}\right)=-\frac{\mathrm{i} \hbar}{2 M c} \frac{1}{\left(r_{b} r_{a}\right)} \frac{M}{\hbar \tilde{k}} \frac{\left|\Gamma\left(-\mathrm{i} \tilde{v}+\tilde{l}_{D}+1\right)\right|^{2}}{\left[\left(2 \tilde{l}_{D}+1\right) !\right]^{2}} \\
& \times \mathrm{e}^{\pi \tilde{v}} M_{-\mathrm{i} \tilde{v}, \tilde{l}_{D}+1 / 2}\left(2 \mathrm{i} \tilde{k} r_{b}\right) M_{\mathrm{i} \tilde{v}, \tilde{l}_{D}+1 / 2}\left(-2 \mathrm{i} \tilde{k} r_{a}\right) .
\end{aligned}
$$

Thus we have

$$
\begin{gathered}
\int_{M c^{2}}^{\infty} \frac{\mathrm{d} E_{D}}{2 \pi \hbar} \operatorname{disc} G\left(r_{b}, r_{a} ; E_{D}>M c^{2}, l_{D}\right) \\
=\frac{1}{2 \pi \hbar} \int_{-\infty}^{\infty} \frac{(\hbar c)^{2} \tilde{k} \mathrm{~d} \tilde{k}}{\sqrt{M^{2} c^{4}+(\hbar c \tilde{k})^{2}}} \operatorname{disc} G\left(r_{b}, r_{a} ; E_{D}>M c^{2}, l_{D}\right) \\
=-\frac{\mathrm{i} \hbar}{2 M c} \frac{1}{\left(r_{b} r_{a}\right)} \int_{-\infty}^{\infty} \mathrm{d} \tilde{k}\left(\frac{E_{D}}{M c^{2}}\right) R_{\tilde{k} l_{D}}(r) R_{\tilde{k} l_{D}}^{*}(r) .
\end{gathered}
$$


From this, we obtain the continuous radial wavefunction of the relativistic dyonium system

$$
\begin{aligned}
& R_{\tilde{k} l_{D}}(r)=\sqrt{\frac{1}{2 \pi}} \frac{1}{\left[1+\left(c \hbar \tilde{k} / M c^{2}\right)^{2}\right]^{1 / 2}} \frac{\left|\Gamma\left(-\mathrm{i} \tilde{\nu}+\tilde{l}_{D}+1\right)\right|}{\left(2 \tilde{l}_{D}+1\right) !} \mathrm{e}^{\pi \tilde{v} / 2} M_{\mathrm{i} \tilde{\nu}, \tilde{l}_{D}+1 / 2}(-2 \mathrm{i} \tilde{k} r) \\
&= \sqrt{\frac{1}{2 \pi}} \frac{1}{\left[1+\left(c \hbar \tilde{k} / M c^{2}\right)^{2}\right]^{1 / 2}} \frac{\left|\Gamma\left(-\mathrm{i} \tilde{\nu}+\tilde{l}_{D}+1\right)\right|}{\left(2 \tilde{l}_{D}+1\right) !} \\
& \times \mathrm{e}^{\pi \tilde{v} / 2} \mathrm{e}^{\mathrm{i} k r}(-2 \mathrm{i} \tilde{k} r)^{\tilde{l}_{D}+1} M\left(-\mathrm{i} \tilde{v}+\tilde{l}_{D}+1,2 \tilde{l}_{D}+2 ;-2 \mathrm{i} \tilde{k} r\right) .
\end{aligned}
$$

It is easy to check that the result is in accordance with the non-relativistic wavefunction when we take the non-relativistic limit.

\section{Concluding remarks}

In this paper, Kleinert's relativistic path integral with the magnetic interaction is studied. As an application, we have calculated the path integral of the relativistic dyonium system. The result is separated into the monopole harmonics and the radial path integral, and the radial fixed-energy amplitude is found in closed form. The dyonium case serves as a prototype of the path integral for a relativistic particle in arbitrary potentials. From this problem, we see the merits of the path integral approach in the treatment of the magnetic interactions. Under the Dirac's charge quantization condition, the magnetic interaction just involves the boundary integration. This procedure is something like that of the path integral treatment of the ABeffect [19] in which the magnetic interaction is reduced to the sum of the topological winding number.

It is our hope that our studies will help to achieve the ultimate goal of obtaining a comprehensive and complete description of quantum mechanics from the point of view of fluctuating paths.

\section{Acknowledgments}

The authors are grateful to Dr M C Chang and X Xu for helpful discussions. The work is supported by the National Science Council of the ROC under contract number NSC88-2811M-009-0015.

\section{References}

[1] Grosche C and Steiner F 1998 Handbook of Feynman Path Integrals (Springer Tracts in Modern Physics vol 145) (Berlin: Springer)

[2] Kleinert H 1996 Phys. Lett. A 21215

[3] Barut A O, Schneider C K E and Wilson R 1979 J. Math. Phys. 202244

[4] Bose S K 1985 J. Phys. A: Math. Gen. 181289

[5] Jackiw R 1980 Ann. Phys. 129183

[6] Schwinger J 1969 Science 165757

[7] D'Hoker E and Vinet L 1985 Nucl. Phys. B 26079

[8] Schwinger J, Milton K A, Tsai W Y, DeRaad L L Jr and Clark D C 1976 Ann. Phys. 101451

[9] Kleinert H 1986 Phys. Lett. A 116201

[10] Dürr H and Inomata A 1985 J. Math. Phys. 262231

[11] Lin D H 1998 J. Phys. A: Math. Gen. 317577

[12] Fujikawa K 1996 Prog. Theor. Phys. 96863

Fujikawa K 1997 Nucl. Phys. B $\mathbf{4 8 4} 495$ 
[13] Kleinert H 1999 Phys. Lett. A 252277

(Kleinert H 1998 Preprint quant-ph/9807073)

[14] Kleinert H 1992 Int. J. Mod. Phys. A 74693

Kleinert H 1990 Phys. Lett. B 246127

Kleinert H 1992 Phys. Lett. B 293168

[15] Duru I H and Kleinert H 1979 Phys. Lett. B 84185

Duru I H and Kleinert H 1982 Fortschr. Phys. 30401

[16] Kleinert H 1995 Path Integrals in Quantum Mechanics, Statistics and Polymer Physics (Singapore: World Scientific)

[17] Ho R and Inomata A 1982 Phys. Rev. Lett. 48231

[18] Gradshteyn I S and Ryzhik I M 1994 Table of Integrals, Series and Products (New York: Academic)

[19] Lin D H 1998 J. Phys. A: Math. Gen. 314785 